\title{
Correction: RANBP1 promotes colorectal cancer progression by regulating pre-miRNA nuclear export via a positive feedback loop with YAP
}

Dandan Zheng, Meng Cao, Siyu Zuo, Xin Xia, Chunchun Zhi, Yanbing Lin, Sitong Deng and Xiaoqin Yuan (D)

(C) The Author(s), under exclusive licence to Springer Nature Limited 2021

Oncogene (2022) 41:1070; https://doi.org/10.1038/s41388-021-02152-2

Correction to: Oncogene https://doi.org/10.1038/s41388-02102036-5

The primary error occurs in Fig. $2 \mathrm{M}$ "RKO si-1" panel, this cell invasion image was actually from the transwell of Fig. $2 \mathrm{M}$ "RKO si$3^{\prime \prime}$ taken under different fields of the microscope. The mistake was introduced during the final assembly of Fig. $2 \mathrm{M}$. This error does not influence the interpretations and conclusions for Fig. $2 \mathrm{M}$ or the work as a whole. The corrected version of Fig. $2 \mathrm{M}$ where the original Fig. $2 \mathrm{M}$ "RKO si-1" was replaced with the right one is shown below.

M

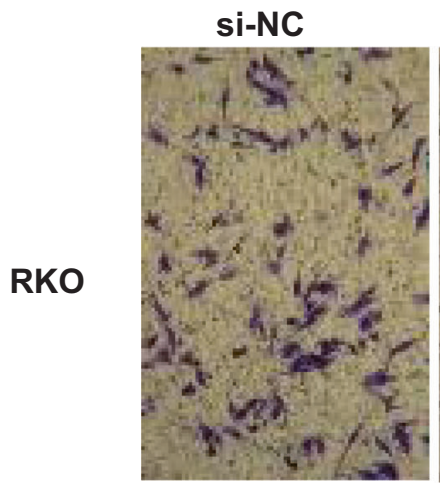

si-1

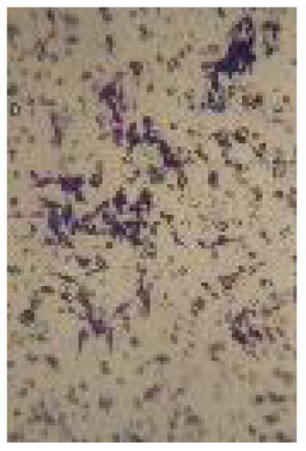

si-3

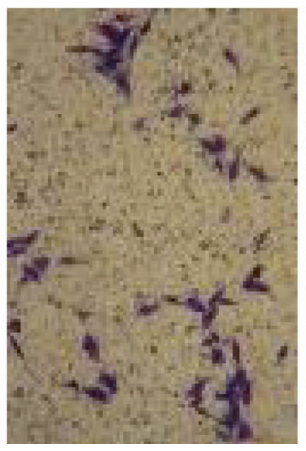

pcDNA3.1

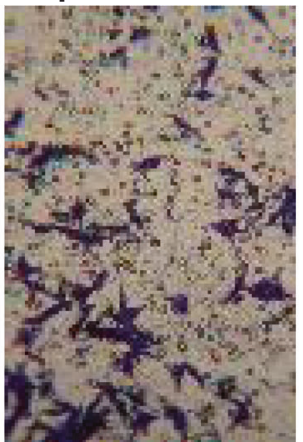

RANBP1

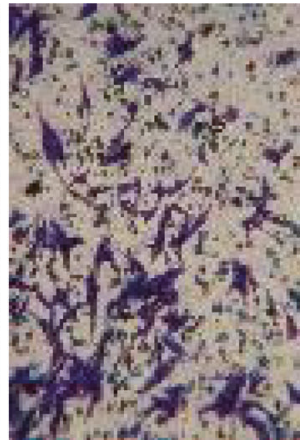

The original article has been corrected. 